\title{
Genicular artery embolization as a novel treatment for mild to moderate knee osteoarthritis: protocol design of a randomized sham-controlled clinical trial
}

\author{
T. A. van Zadelhoff ${ }^{*}$, A. Moelker' ${ }^{1}$ S. M. A. Bierma-Zeinstra' ${ }^{2}$ P. K. Bos ${ }^{3}$, G. P. Krestin ${ }^{1}$ and E. H. G. Oei ${ }^{1}$
}

\begin{abstract}
Introduction: Knee osteoarthritis is a common disease with pain as the most prevalent symptom. Previous cohort studies have shown genicular artery embolization to reduce pain symptoms in patients with mild to moderate knee osteoarthritis. Patients resistant to conservative therapy but not eligible yet for surgical treatment due to young age or comorbidities may profit from an effective and sustained pain reduction treatment. This study is a randomized sham-controlled trial to evaluate the efficacy of genicular artery embolization in patients with knee osteoarthritis.

Methods and analysis: Fifty-eight patients with mild-to-moderate knee osteoarthritis will be recruited and randomly allocated to the treatment or control group in a 1:1 ratio. Participants in the treatment group will undergo genicular artery embolization. Patients in the control group will undergo sham treatment. Outcome measurements will be assessed at baseline and after 1, 4, 8, and 12 months with questionnaires, pressure pain threshold testing, and MR imaging. The MR imaging protocol is designed to (semi)quantitatively assess osteoarthritis in the knee joint. The primary outcome is the change from baseline of the Knee injury and Osteoarthritis Outcome Score (KOOS) pain subscale after 4 months. Secondary outcomes include change in osteoarthritis-related questionnaires, pressure pain threshold, and OA-related MRI features, particularly synovitis and bone marrow lesions.
\end{abstract}

Ethics and dissemination: This trial will determine the efficacy of genicular artery embolization compared to a sham treatment. This is of importance to assess before proceeding to larger-scale efficiency studies and, ultimately, implementing this treatment into day to day clinical practice.

Trial registration: ClinicalTrials.gov NCT03884049. Registered on 21 March 2019

\section{Introduction}

Knee osteoarthritis (OA) is the most common joint disorder and a leading cause of morbidity worldwide, ranking highly on the list of contributors to disability [1]. OA also induces substantial costs both directly through medical costs as well as indirectly through loss of work

\footnotetext{
* Correspondence: t.vanzadelhoff@erasmusmc.nl

'Department of Radiology and Nuclear Medicine, Erasmus MC, University Medical Center Rotterdam, Rotterdam, The Netherlands

Full list of author information is available at the end of the article
}

productivity [2]. The most common complaint is pain. At early stages, OA is treated with exercise, weight management and topical or oral non-steroidal antiinflammatory drugs (NSAIDs), and add on therapy with a brace and/or intra articular corticosteroid injections [3]. End-stage knee OA is treated surgically with knee joint realignment, unicompartmental or total knee arthroplasty. However, not all patients resistant to conservative therapy are automatically eligible for surgical treatment due to young age or comorbidities.

C The Author(s). 2022 Open Access This article is licensed under a Creative Commons Attribution 4.0 International License, which permits use, sharing, adaptation, distribution and reproduction in any medium or format, as long as you give appropriate credit to the original author(s) and the source, provide a link to the Creative Commons licence, and indicate if changes were made. The images or other third party material in this article are included in the article's Creative Commons licence, unless indicated otherwise in a credit line to the material. If material is not included in the article's Creative Commons licence and your intended use is not permitted by statutory regulation or exceeds the permitted use, you will need to obtain permission directly from the copyright holder. To view a copy of this licence, visit http://creativecommons.org/licenses/by/4.0/ The Creative Commons Public Domain Dedication waiver (http://creativecommons.org/publicdomain/zero/1.0/) applies to the data made available in this article, unless otherwise stated in a credit line to the data. 
Furthermore, some patients choose to not undergo surgical treatment themselves despite increasing pain and limitation [4]. There are currently no effective alternative treatments for this group of patients. It is estimated that 3.6 million Americans belong to this group and this number is projected to increase to 5 million by 2025 [5].

Recent clinical studies have shown an effective pain reduction following genicular artery embolization treatment (GAE), during which periarticular angiogenesis is embolized [6-9]. The observed improvement of pain symptoms, combined with new insights in the pathophysiology of knee OA, offer an opportunity for a novel treatment beneficial to patients in the aforementioned treatment gap. Targeting angiogenesis, which is increased in among others the synovium and the osteochondral junction, potentially reduces pain symptoms [10]. This angiogenesis is thought to be associated with synovitis, osteochondral damage and osteophyte formation. It is also accompanied by nerve ingrowth, innervating tissues that are normally not innervated. Therefore, decreasing angiogenesis through GAE may potentially have a positive effect on symptomatic knee OA.

Okuno et al. used GAE to reduce angiogenesis in 95 patients with knee OA [7]. They found a significant reduction of pain symptoms measured by the Western Ontario and McMaster Universities Osteoarthritis Index (WOMAC) pain scores (0-20 scale) from 12.1 at baseline to 2.6 after 24 months. More recently, in a study of 20 patients, Bagla et al. found a significant reduction of the mean pain visual analog score (VAS) of 76 to 29 on a $0-100$ scale [8]. Although these results show promise of GAE to bridge the treatment gap for a significant proportion of knee OA patients, these previous studies were both prospective non-randomized cohort studies. Hence, it is unknown how much of the observed effect is attributable to the placebo effect. It is well known that the placebo effect plays a major role in knee OA treatment and that the type of treatment is a determinant for the magnitude of the placebo effect, which has been shown to be larger for invasive treatments [11]. To rule out the placebo effect and determine the true efficacy of GAE, a randomized controlled trial with a sham control group is mandatory. Furthermore, to investigate changes induced by GAE and understand how angiogenesis is tied in with knee OA symptoms, imaging is warranted.

Therefore, the main objective is to assess whether genicular artery embolization for patients with symptomatic knee OA results in significant pain reduction after 4 months compared to sham treatment. A secondary aim is to obtain further insight into the role of angiogenesis in knee OA pathophysiology using advanced magnetic resonance imaging (MRI). A tertiary aim is to investigate whether pain sensitization is influenced by GAE using pressure point threshold testing.

\section{Methods and analysis Study design}

This study is a single-center, double blind randomized sham controlled trial. It will be performed at the Erasmus MC, University Medical Center Rotterdam, the Netherlands.

\section{Study population and recruitment}

This is a study with multi-center recruitment from orthopedic outpatient clinics of the Erasmus MC and hospitals in the region. Written informed consent will be obtained by a clinical researcher prior to enrollment in the trial. The study population consists of patients age $\geq$ 18 years with symptomatic, mild-to-moderate radiographic knee OA (Kellgren and Lawrence (KL) grade 13) [12] resistant to conservative therapy. In order to be eligible for participation, we defined the following inand exclusion criteria:

\section{Inclusion criteria}

- Age $\geq 18$ years

- Knee pain for a duration of $\geq 6$ months

- Knee pain (numeric rating scale $\geq 4$ to $\leq 8$ ) on at least half of the days in the preceding month at time of inclusion

- Insufficient response to conservative treatment for at least 6 months

- Radiographic knee osteoarthritis (KL grade 1-3)

\section{Exclusion criteria}

- Contra-indications for MRI (e.g., metallic foreign bodies, etc.)

- Contra-indications for angiography (e.g., coagulopathy)

- Previous surgical treatment for knee osteoarthritis (e.g., high tibial osteotomy), excluding knee arthroscopy

- Musculoskeletal co-morbidity (e.g., rheumatoid arthritis or gout) potentially masking the effect of GAE

- Renal insufficiency, determined with a blood sample test $\left(\right.$ GFR $\left.<30 \mathrm{ml} / \mathrm{min} / 1.73 \mathrm{~m}^{2}\right)$

- Known allergy to contrast agents;

- Known allergies to barium sulfate, 3aminopropyltrialkoxysilane, polyphosphazene

- Women who are pregnant or lactating

- Intermittent claudication of affected limb

- Intra-articular injections in the ipsilateral knee less than 6 months ago 
- On the waiting list for joint replacement surgery

- Amitriptyline usage

- Insufficient command of the Dutch language

- Legally incompetent adults

Participation in this trial is voluntary and patients can stop at any time without providing a reason. Patients withdrawn from the trial will be invited to fill in the questionnaires from home if they are not undergoing any other form of treatment for knee OA.

\section{Randomization, allocation concealment, and blinding}

Patients will be randomized 1:1 using a random block size strategy with block sizes of 4 and 6 . Randomization is performed using an online randomization tool (ALEA clinical: https://www.aleaclinical.eu/). The clinical researcher, blinded for randomization, will conduct the MRI scans, evaluate patients during follow-up, and will enter the patient data into an online database. The interventional radiologist who will perform the procedure will be automatically informed about the randomization result by email. The allocation will only be sent to the interventional radiologist and a third party within the department who safeguards the randomization code. All others, including the clinical researcher, treating orthopedic surgeon, and patients, will be blinded tot group allocation.

\section{Interventions}

To ensure patients will be effectively blinded, several precautions are taken. The personnel in the intervention suite will be instructed not to mention the group the patient is allocated to or anything else that could compromise blinding status of participants. Patients will lie in supine position and wear a noise-canceling headphone with music to distract them from the procedure and surrounding sounds. The patient's line of sight on the intervention working field and imaging monitors will be blocked by a surgical drape. The skin around the knee is cooled using ice packs to minimize non-target embolization to the skin vasculature. The groin area will be anaesthetized with $2 \%$ lidocaine $10 \mathrm{ml}$.

\section{GAE procedure}

Patients allocated to the treatment group will receive GAE treatment, performed by an interventional radiologist experienced in vascular embolization procedures.

Following local anesthesia, an antegrade 4 French (Fr) catheter will be inserted into the common femoral artery. Initial digital subtraction angiography targeted on the vessels around the knee will be performed using Iodixanol $320 \mathrm{mg} \mathrm{I} / \mathrm{ml}$ (Visipaque 320, GE Healthcare, Chicago, IL, USA). Culprit vessels will be catheterized using a $1.8 \mathrm{Fr}$ microcatheter with micro guidewire and embolized using Embozene Microspheres $75 \mu \mathrm{m}$ or $100 \mu \mathrm{m}$ (Varian Medical Systems, Palo Alto, CA, USA) dissolved in $20 \mathrm{ml}$ of Visipaque 320 until stasis of flow is achieved. When no evident culprit vessel is identified, the distal branch of the genicular artery corresponding with the most painful location is embolized. After finishing the embolization procedure, the sheath will be removed, and the puncture location is manually compressed for at least $10 \mathrm{~min}$ after which patient is immobilized for at least $3 \mathrm{~h}$, followed by discharge. The procedure time varies between 1 and $2 \mathrm{~h}$.

\section{Sham procedure}

In the sham group, the patient setup is exactly the same as in the treatment group. After anesthesia, a small incision will be made mimicking the incision in the GAE group. The interventional radiologist will pretend to insert a catheter in the femoral artery and perform angiography and embolization. The common femoral artery will not be punctured. The $\mathrm{C}$-arm of the angiography system and the table will be moved during the sham procedure mimicking the actual procedure; however, no images are actually made, so there is no unnecessary radiation exposure. The sham procedure will take approximately as long as the actual intervention.

After both procedures, the site of incision will be manually compressed for $10 \mathrm{~min}$ after which the patient is immobilized for at least $3 \mathrm{~h}$ followed by discharge. Before discharge, blinding for group allocation will be tested in all patients by asking in what group they think they were allocated to. They have 3 options: "intervention group," "sham group," and "I don't know." This timing has been chosen since testing later on in the trial could potentially be influenced by efficacy and side effects of the treatment [13].

\section{Co-interventions}

Data on the usage of co-interventions will be collected at every follow-up visit through an interview. Patients will be encouraged not to start any new conservative treatments they were not receiving at baseline. Besides no new pharmacological treatment, this also includes no new corticosteroid injections, physiotherapy, or the usage of a brace. They can, however, increase or decrease any pharmacological treatment they were receiving based on their complaints, continue an already ongoing physiotherapy treatment, or keep wearing a brace if they were using one. This information will be recorded.

\section{Outcome measurements}

Outcome measurements will be recorded at baseline and $1,4,8$, and 12 months after the procedure. The primary outcome is change of the knee injury and osteoarthritis 
outcome score (KOOS [14]) pain subscale score between baseline and 4 months follow-up. Secondary outcomes are the change of pain subscale score between baseline and 1,8 , and 12 months. Other secondary outcomes are the change of the remaining dimensions of the KOOS (symptoms, daily living, sport and recreation and quality of life), additional questionnaires, and the pressure pain threshold after 1, 4, 8, and 12 months.

Changes on MRI will be compared between baseline and after 1 and 4 months. All clinical trial study activities are summarized in Table 1.

\section{Questionnaires}

For the main outcome, the KOOS questionnaire is used [14]. This questionnaire consists of 42 questions divided into 5 subscales: pain (primary outcome), other symptoms, function in daily living (ADL), function in sport and recreation, and knee-related quality of life (QOL). Other questionnaires used are the Intermittent and constant osteoarthritis pain (ICOAP) [15], painDETECT [16], EuroQol 5 dimensions 5 levels (EQ-5D-5L) quality of life questionnaire [17], and a VAS $(0-100 \mathrm{~mm})$ score for pain, stiffness, and swelling. At the 12-month followup point, patients will be asked about the perceived effect of the treatment using the global perceived effect questionnaire [18]. All patients will fill in the questionnaires autonomously, but if some questions are not understood, they can ask the clinical researcher for assistance.

\section{Pressure pain threshold testing}

At baseline and each follow-up visit, pressure pain threshold testing (PPT) will be performed using an algometer with a tip of $1 \mathrm{~cm}^{2}$ (Biometrics, MicroFET 2, Almere, The Netherlands). Measurements will be performed with the patient's knee in $30^{\circ}$ of flexion. The sites of measurements are at the medial and lateral joint space $2 \mathrm{~cm}$ medial and lateral to the inferior edge of patella, $2 \mathrm{~cm}$ above the superior border of the patella, and a peripheral site at the center of the brachioradialis muscle at the contralateral side from the treated knee. Pressure of gradually increasing intensity $\left(10 \mathrm{~N} \cdot \mathrm{s}^{-1}\right)$ is applied. The patient is instructed to say "stop" at the moment the sensation switches from pressure to painful. These measurements are repeated three times and are averaged to determine the pressure pain threshold. The sites are marked such that repeat measurements will be at the same location. Before measurements start, a test

Table 1 Clinical trial study activities overview

\begin{tabular}{|c|c|c|c|c|c|c|c|}
\hline \multirow[b]{3}{*}{ Timepoint $^{* *}$} & \multicolumn{7}{|l|}{ Study period } \\
\hline & \multirow{2}{*}{$\begin{array}{l}\text { Enrolment/baseline } \\
-2 \text { weeks }\end{array}$} & \multirow{2}{*}{$\begin{array}{l}\text { Intervention } \\
0\end{array}$} & \multicolumn{4}{|l|}{ Post-allocation } & \multirow{2}{*}{$\begin{array}{l}\text { Close-out } \\
12 \text { months }\end{array}$} \\
\hline & & & Post-intervention & 1 month & 4 months & 8 months & \\
\hline \multicolumn{8}{|l|}{ Enrolment: } \\
\hline Eligibility screen & $\mathrm{x}$ & & & & & & \\
\hline Informed consent & $\mathrm{x}$ & & & & & & \\
\hline Randomization & $\mathrm{x}$ & & & & & & \\
\hline Allocation & $\mathrm{x}$ & & & & & & \\
\hline \multicolumn{8}{|l|}{ Interventions: } \\
\hline Genicular artery embolization & & $\mathrm{x}$ & & & & & \\
\hline Sham procedure & & $\mathrm{x}$ & & & & & \\
\hline \multicolumn{8}{|l|}{ Assessments: } \\
\hline Blood examination & $\mathrm{x}$ & & & & & & \\
\hline Usage of co-interventions & $x$ & & & $\mathrm{x}$ & $\mathrm{x}$ & $\mathrm{x}$ & $\mathrm{x}$ \\
\hline VAS pain & $x$ & & & $x$ & $x$ & $x$ & $\mathrm{x}$ \\
\hline KOOS questionnaire & $x$ & & & $x$ & $x$ & $x$ & $x$ \\
\hline ICOAP questionnaire & $\mathrm{x}$ & & & $\mathrm{x}$ & $x$ & $\mathrm{x}$ & $\mathrm{x}$ \\
\hline painDETECT questionnaire & $x$ & & & $x$ & $x$ & $x$ & $x$ \\
\hline EQ-5D-5L questionnaire & $\mathrm{x}$ & & & $\mathrm{x}$ & $\mathrm{x}$ & $x$ & $x$ \\
\hline Global perceived effect questionnaire & & & & & & & $x$ \\
\hline PPT measurement & $\mathrm{x}$ & & & $x$ & $x$ & $\mathrm{x}$ & $x$ \\
\hline MRI examination & $\mathrm{x}$ & & & $x$ & $x$ & & \\
\hline Adverse event assessment & & & $\mathrm{x}$ & $x$ & $\mathrm{x}$ & $\mathrm{x}$ & $\mathrm{x}$ \\
\hline Concealment of allocation assessment & & & $\mathrm{x}$ & & & & \\
\hline
\end{tabular}


measurement will be done at a site not used for actual measurements for patients to get familiar with the measurements. Measurements are repeated 3 times and the average will be considered the pressure pain threshold. This method to determine the PPT has a high reliability with intra class correlation coefficients of 0.95-0.97 [19]. A cutoff limit of $120 \mathrm{~N} / \mathrm{cm}^{2}$ was implemented to prevent tissue damage.

\section{MRI examination}

All patients will undergo MRI at baseline and at 1 and 4 months after treatment. Images will be acquired using a 3.0-T MR system with $70 \mathrm{~cm}$ bore (Signa Premier, GE, Chicago, IL, USA) equipped with an 18-channel dedicated knee coil (GE, Chicago, IL, USA). The MRI protocol consists of Double Echo Steady State (DESS), 3D PD (proton density) weighted fast spin echo, axial and sagittal T2 fat saturated (FS), sagittal MAGnetic resonance Image Compilation (MAGiC) synthetic imaging, dynamic contrast enhanced (DCE) MRI using DIfferential Subsampling with Cartesian Ordering (DISCO), and post contrast T1 weSPGR pulse sequences. During the DCEMRI, $0.1 \mathrm{ml} / \mathrm{kg}$ gadovist (Bayer, Leverkusen, Germany) followed by $15 \mathrm{ml}$ of saline will be administered at an injection speed of $1.0 \mathrm{ml} / \mathrm{s}$ using a contrast injector (GE, Chicago, IL, USA). This protocol was designed to assess the knee tissues semi quantitatively using the MRI Osteoarthritis Knee Score index (MOAKS [20]) and quantitatively using DCE-MRI and post contrast T1 acquisitions as well as T2-mapping of the articular cartilage using the MAGiC acquisitions.

\section{Adverse events and the data safety monitoring board}

Adverse events (AE) are defined as any undesirable experience occurring to a subject during the study period, whether or not considered related to the intervention. One day after the procedure, the patients will be contacted and actively screened for the occurrence of any adverse events. Patients will be asked to report any changes in their health status. At every follow-up visit, patients will be actively screened for the occurrence of any $\mathrm{AE}$.

Possible adverse events related to GAE are as follows: transient discoloration in the region of embolization due to non-target embolization or nerve damage due to nontarget embolization. Only patients in the intervention group are subjected to these risks. All patients will receive local anesthesia and a small incision in the groin and are therefore subject to the risk of access-site hematoma and access-site infection. All patients undergo MRI with contrast agent injection and are therefore at risk for an allergic reaction to gadolinium.

After including 10 patients, a data and safety monitoring board (DSMB) will perform a safety analysis. The
DSMB consists of an interventional radiologist, an orthopedic surgeon, and a biostatistician. The aim of this committee is to protect the interests of patients enrolled in this study and to evaluate the safety of the investigational intervention used in this trial. (S)AE's and pain scores will be used to test if the intervention is deemed safe enough to continue the trial. The local ethical board will receive a report and will decide if the trial can continue. The members of this DSMB are independent of the trial. They were not involved in the creation of the protocol or have any competing interest.

Additionally, the trial conduct will be audited every 6 months by an independent auditor from the Department of Radiology \& Nuclear Medicine of Erasmus MC.

\section{Sample size calculation}

Calculations are partly based on the previous observational studies by Okuno et al. [6, 7]. Despite a possible placebo effect, which account for effect sizes up to 0.5 [21], they found effect sizes of $>5$. For our study, we assumed we would require to demonstrate a large effect size of $>0.8$ (strong effect) compared to sham embolization since GAE still is (minimally) invasive. Assuming this projected effect size of 0.8 , and a standard deviation of 16 for the KOOS (100-0) pain score (estimated from multiple OA studies at Erasmus MC), beta of 0.80 , and alpha of $0.05,48$ patients $(n=24$ per study $\mathrm{arm})$ are required to detect an increase (higher scores indicate less pain) in primary outcome measure of 8 points in the control group (placebo effect) compared to 21 points in the intervention group. The targeted sample size will be 58 to account for approximately $20 \%$ of patients lost to follow-up. We also expect a more homogeneous study population since all included subjects have an NRS pain score $\geq 4$ and $\leq 8$. With this sample size, and in case of lower variation in pain scores than SD of 16 , we expect we could also detect smaller effect sizes between 0.5 (moderate effect) and 0.8 .

\section{Data management}

All outcomes will be entered into OpenClinica Community software (Version: 3.12.2, OpenClinica LLC and collaborators, Waltham, MA, USA, www.OpenClinica. com.), an online password protected electronic data capture system. Data will be collected anonymously. A source document review of randomly selected subjects will take place to ensure correct entering of data. All paper files will be preserved for at least 15 years.

\section{Statistical analysis}

The primary outcome is the difference in KOOS pain subscale change from baseline to 4 months between both groups. Repeated measurements regression analysis for continuous variables will be performed, based on 
intention to treat analysis to determine a difference between groups. Generalized estimating equations (GEE) will be used to enable inclusion of all available measurements in the regression models even if they are correlated. It also allows us to adjust for unequal distribution of prognostic variables over the two groups, which easily can occur in smaller randomized clinical trials. Radiographic severity, pain pressure threshold, and degree of sensitized pain according to painDetect will all be tested for equal distribution and, if more than $10 \%$ different, we will test if this variable changes the estimate with more than $10 \%$. If this is the case, the variable will be included in the adjusted analysis. The adjusted analysis will be the primary analysis, but we will also present the unadjusted analysis. If some of the patients do not receive the treatment as allocated, we will also perform a per protocol analysis where the patients that did not comply with the allocated treatment will be excluded. Repeated measurement regression analysis will also be used for all continuous secondary outcomes: KOOS subscales, VAS, ICOAP, painDETECT, EQ-5D-5L, and PPT scores.

\section{Discussion}

GAE is a novel intervention for patients with symptomatic, mild-to-moderate knee OA [6,7]. Initial results from cohort studies show promising outcomes with up to 4 years of pain improvement but the studies lacked a control group [5]. This randomized trial is designed to determine the efficacy of this treatment compared to a sham treatment. Sham controlled surgical trials for knee $\mathrm{OA}$ and degenerative meniscal tear treatment have had great impact on current insight in treatment of symptomatic knee OA. Not until sham controlled studies demonstrated no difference in pain reduction between surgery and sham surgery was the procedure removed from guidelines $[21,22]$. We firmly believe that, before introducing promising new treatments, these have to be evaluated against control groups. For surgical therapies, this involves the use of sham treatment.

In our initial protocol, we planned for the sham group to undergo angiography of the genicular vessels without embolization in order to visualize and compare the angiogenesis in both groups. However, after consulting with the local ethical committee, this was deemed too invasive as a sham intervention for patients who already had knee complaints and was not necessary to achieve the primary objective. Therefore, we changed the sham treatment to be an incision in the groin without a catheter entering the femoral artery. This less invasive form of a sham procedure is a challenge with regard to blinding. Patients must be under the impression that a catheter is actually entering the arteries and that the interventional radiologist is performing the procedure. Using noise-canceling headphones so patients cannot hear their surroundings, using the $\mathrm{C}$-arm to mimic angiography, and giving patients in the sham group the same post procedural care as the intervention group are examples of how we maintain adequate blinding. The upside of changing the sham procedure to this less invasive method is that patients will not be exposed to any unnecessary additional risk associated with angiography. To assess whether we succeeded to keep the intervention blinded for the patient, we will ask them for their own perception of group allocation after the treatment.

Several research groups are currently evaluating the outcome of GAE. Recently, results of non-randomized pilot studies have been published showing promising results; however, the authors also state the importance of testing GAE in a randomized controlled trial $[8,9]$. Because GAE shows potential but is also invasive and incurs additional costs, a thorough evaluation is warranted before being introduced into clinical practice.

We are aware of one published protocol describing a similar trial [23]. We believe that multiple trial outcomes will strengthen the evidence upon which we can draw a conclusion on the efficacy of GAE. There are some differences to the aforementioned study protocol. Using a sophisticated MRI protocol including high-resolution DCE-MRI and testing pain pressure thresholds, we aim to expand the knowledge on the working mechanism of GAE. DCE-MRI provides quantitative measures of perfusion. This can be used to monitor changes occurring due to the alteration of angiogenesis by GAE [24]. It is suggested that DCE-MRI derived parameters are more sensitive to treatment response and associate stronger with pain changes than synovial volume [25]. If pain reduction due to GAE is truly achieved through reduction of synovitis, the perfusion parameters in the synovium should decrease commensurate with clinical improvement.

\section{Trial status}

The current protocol version is 1.6 , dated at 31 July 2020 and is approved by the local research ethics committee. As of 26 April 2020, we have included 40 patients and aim to finish inclusion in the first half of 2021. After 10 inclusions, there was a safety interim analysis by an independent data safety monitoring board. They compared adverse events occurring in both groups to confirm no unacceptable (serious) adverse events occurred in either group. The DSMB concluded the trial was safe to continue.

Furthermore, Bagla et al. noticed a reduced amount of neurogenic complications when they used $100 \mu \mathrm{m}$ 
embolization particles compared to $75 \mu \mathrm{m}$ [8]. Therefore, we added the $100 \mu \mathrm{m}$ variant as a usable embolic agent.

\section{Abbreviations}

OA: Osteoarthritis; NSAID: Non-steroid anti-inflammatory drugs;

GAE: Genicular artery embolization; KL: Kellgren and Lawrence; Fr: French; WOMAC: Western Ontario and McMaster Universities osteoarthritis Index VAS: Visual analog score; ICOAP: Intermittent and constant osteoarthritis pain; MRI: Magnetic resonance imaging; DCE: Dynamic contrast enhanced; MOAKS: MRI osteoarthritis knee score; KOOS: Knee injury and Osteoarthritis Outcome Score; PPT: Pressure pain threshold; MAGiC: MAGnetic resonance Image Compilation; DISCO: DIfferential Subsampling with Cartesian Ordering; weSPGR: Water excitation spoiled gradient recalled; GEE: Generalized estimating equations; DSMB: Data and safety monitoring board

\section{Authors' contributions}

EHGO and AM conceived the trial and obtained funding. All authors helped design the protocol. All authors contributed to the draft writing or editing of the manuscript. TVZ enrolls patients and collects all the data. AM performs all the interventions. All authors read an approved the final manuscript.

\section{Funding}

This trial was sponsored by COOK medical, Stichting Coolsingel, and the Erasmus MC Efficiency Program. The sponsors had no role in the design of the trial.

\section{Availability of data and materials}

The datasets used and/or analyzed during the current study are available from the corresponding author on reasonable request.

\section{Declarations}

\section{Communication of trial results and sharing data}

Trial results will be disseminated in scientific publications. There are no plans for sharing any data.

\section{Ethics approval and consent to participate}

The study protocol has been approved by the medical ethical committee of the Erasmus MC (CPB/410817). Written informed consent will be obtained from all participants prior to enrolment in the study. Any substantial modifications to the protocol will be submitted to the medical ethical committee for approval.

\section{Consent for publication}

Not applicable, there is no patient data.

\section{Competing interests}

The authors declare that they have no competing interests.

\section{Author details}

'Department of Radiology and Nuclear Medicine, Erasmus MC, University Medical Center Rotterdam, Rotterdam, The Netherlands. ${ }^{2}$ Department of General Practice, Erasmus MC, University Medical Center Rotterdam, Rotterdam, The Netherlands. ${ }^{3}$ Department Orthopaedic surgery, Erasmus MC, University Medical Center Rotterdam, Rotterdam, The Netherlands.

Received: 11 May 2021 Accepted: 15 December 2021

Published online: 08 January 2022

\section{References}

1. Cross M, Smith E, Hoy D, Nolte S, Ackerman I, Fransen M, et al. The global burden of hip and knee osteoarthritis: estimates from the Global Burden of Disease 2010 study. Annals of the Rheumatic Diseases. 2014;73(7):1323-30.

2. Salmon JH, Rat AC, Achit H, Ngueyon-Sime W, Gard C, Guillemin F, et al. Health resource use and costs of symptomatic knee and/or hip osteoarthritis. Osteoarthritis Cartilage. 2019;27(7):1011-7. https://doi.org/10.1 016/j.joca.2019.03.005.

3. McAlindon TE, Bannuru RR, Sullivan MC, Arden NK, Berenbaum F, BiermaZeinstra SM, et al. OARSI guidelines for the non-surgical management of knee osteoarthritis. Osteoarthritis and cartilage. 2014;22(3):363-88. https:// doi.org/10.1016/j.joca.2014.01.003.
4. Jacobson AF, Myerscough RP, Delambo K, Fleming E, Huddleston AM, Bright $\mathrm{N}$, et al. Patients' perspectives on total knee replacement. Am J Nurs. 2008;108(5):54-63 quiz -4.

5. London NJ, Miller LE, Block JE. Clinical and economic consequences of the treatment gap in knee osteoarthritis management. Medical hypotheses. 2011;76(6):887-92. https://doi.org/10.1016/.mehy.2011.02.044.

6. Okuno Y, Korchi AM, Shinjo T, Kato S. Transcatheter arterial embolization as a treatment for medial knee pain in patients with mild to moderate osteoarthritis. Cardiovasc Intervent Radiol. 2015;38(2):336-43. https://doi. org/10.1007/s00270-014-0944-8.

7. Okuno Y, Korchi AM, Shinjo T, Kato S, Kaneko T. Midterm clinical outcomes and MR imaging changes after transcatheter arterial embolization as a treatment for mild to moderate radiographic knee osteoarthritis resistant to conservative treatment. J Vasc Interv Radiol. 2017;28(7):995-1002. https://doi. org/10.1016/j.jvir.2017.02.033.

8. Bagla S, Piechowiak R, Hartman T, Orlando J, Del Gaizo D, Isaacson A. Genicular artery embolization for the treatment of knee pain secondary to osteoarthritis. Journal of Vascular and Interventional Radiology. 2020;31(7): 1096-102. https://doi.org/10.1016/j.jvir.2019.09.018.

9. Landers S, Hely R, Page R, Maister N, Hely A, Harrison B, Gill S. Genicular Artery Embolization to Improve Pain and Function in Early-Stage Knee Osteoarthritis-24-Month Pilot Study Results. J Vasc Interv Radiol. 2020;31(9): 1453-1458. https://doi.org/10.1016/j.jvir.2020.05.007.

10. Mapp PI, Walsh DA. Mechanisms and targets of angiogenesis and nerve growth in osteoarthritis. Nature reviews Rheumatology. 2012;8(7):390-8. https://doi.org/10.1038/nrrheum.2012.80.

11. Zhang W, Robertson J, Jones AC, Dieppe PA, Doherty M. The placebo effect and its determinants in osteoarthritis: meta-analysis of randomised controlled trials. Ann Rheum Dis. 2008;67(12):1716-23. https://doi.org/10.113 6/ard.2008.092015.

12. Kellgren JH, Lawrence JS. Radiological assessment of osteo-arthrosis. Annals of the rheumatic diseases. 1957;16(4):494-502. https://doi.org/10.1136/ard.16.4.494.

13. Kolahi J, Bang H Fau-Park J, Park J. Towards a proposal for assessment of blinding success in clinical trials: up-to-date review. (1600-0528 (Electronic)).

14. Roos EM, Roos HP, Lohmander LS, Ekdahl C, Beynnon BD. Knee Injury and Osteoarthritis Outcome Score (KOOS)--development of a self-administered outcome measure. J Orthop Sports Phys Ther. 1998;28(2):88-96. https://doi. org/10.2519/jospt.1998.28.2.88.

15. Hawker GA, Davis AM, French MR, Cibere J, Jordan JM, March L, et al. Development and preliminary psychometric testing of a new OA pain measure - an OARSI/OMERACT initiative. Osteoarthritis and Cartilage. 2008; 16(4):409-14. https://doi.org/10.1016/j.joca.2007.12.015.

16. Freynhagen $R$, Baron R, Gockel U, Tölle TR. painDETECT: a new screening questionnaire to identify neuropathic components in patients with back pain. Curr Med Res Opin. 2006;22(10):1911-20. https://doi.org/10.1185/03 $0079906 \times 132488$.

17. Herdman M, Gudex C, Lloyd A, Janssen M, Kind P, Parkin D, Bonsel G, Badia $X$. Development and preliminary testing of the new five-level version of EQ5D (EQ-5D-5L). Qual Life Res. 2011;20(10):1727-36. https://doi.org/10.1007/ s11136-011-9903-X.

18. Hudak PL, Wright JG. The characteristics of patient satisfaction measures. Spine (Phila Pa 1976). 2000;25(24):3167-77. https://doi.org/10.1097/ 00007632-200012150-00012.

19. Mutlu EK, Ozdincler AR. Reliability and responsiveness of algometry for measuring pressure pain threshold in patients with knee osteoarthritis. J Phys Ther Sci. 2015;27(6):1961-5. https://doi.org/10.1589/jpts.27.1961.

20. Hunter DJ, Guermazi A, Lo GH, Grainger AJ, Conaghan PG, Boudreau RM, et al. Evolution of semi-quantitative whole joint assessment of knee OA: MOAKS (MRI Osteoarthritis Knee Score). Osteoarthritis Cartilage. 2011;19(8): 990-1002. https://doi.org/10.1016/j.joca.2011.05.004.

21. Moseley JB, O'Malley K, Petersen NJ, Menke TJ, Brody BA, Kuykendall DH, et al. A controlled trial of arthroscopic surgery for osteoarthritis of the knee. New England Journal of Medicine. 2002;347(2):81-8. https://doi.org/10.1056/NEJMoa013259.

22. Zhang W, Nuki G, Moskowitz RW, Abramson S, Altman RD, Arden NK, et al. OARSI recommendations for the management of hip and knee osteoarthritis: part III: changes in evidence following systematic cumulative update of research published through January 2009. Osteoarthritis Cartilage. 2010;18(4):476-99. https://doi.org/10.1016/j.joca.2010.01.013.

23. Landers S, Hely A, Harrison B, Maister N, Hely R, Lane SE, et al. Protocol for a single-centre, parallel-arm, randomised controlled superiority trial evaluating the effects of transcatheter arterial embolisation of abnormal knee 
neovasculature on pain, function and quality of life in people with knee osteoarthritis. BMJ Open. 2017;7(5):e014266. https://doi.org/10.1136/ bmjopen-2016-014266.

24. Boesen M, Kubassova O, Sudoł-Szopińska I, Maas M, Hansen P, Nybing JD, Oei EH, Hemke R, Guermazi A. MR Imaging of Joint Infection and Inflammation with Emphasis on Dynamic Contrast-Enhanced MR Imaging. PET Clin. 2018;13(4):523-550. https://doi.org/10.1016/j.cpet.2018.05.007.

25. Gait AD, Hodgson R, Parkes MJ, Hutchinson CE, O'Neill TW, Maricar N, et al. Synovial volume vs synovial measurements from dynamic contrast enhanced MRI as measures of response in osteoarthritis. Osteoarthritis Cartilage. 2016;24(8):1392-8. https://doi.org/10.1016/j.joca.2016.03.015.

\section{Publisher's Note}

Springer Nature remains neutral with regard to jurisdictional claims in published maps and institutional affiliations.

Ready to submit your research? Choose BMC and benefit from:

- fast, convenient online submission

- thorough peer review by experienced researchers in your field

- rapid publication on acceptance

- support for research data, including large and complex data types

- gold Open Access which fosters wider collaboration and increased citations

- maximum visibility for your research: over $100 \mathrm{M}$ website views per year

At BMC, research is always in progress.

Learn more biomedcentral.com/submissions 